\title{
Should sedation holidays be given in the paediatric intensive care unit?
}

Although sedation interruption in the intensive care unit (ICU) is recommended in the literature for adults, its place in paediatrics has been much debated. The arguments for not using sedation interruption protocols include the danger of extubation, ventilatorassociated pneumonia, safety, comfort and, most importantly, synchronisation with the ventilator. There are also conflicting reports and a poor evidence base for the use of this strategy, with only two published randomised controlled trials comparing daily sedation interruption and protocolised sedation. An article by Vet et al. ${ }^{[1]}$ presented the results of a multicentre, randomised controlled trial in the Netherlands on this much-debated issue. The aim of their study was to compare daily sedation interruption plus protocolised sedation (DSI + PS) with protocolised sedation (PS) only in critically ill children. They excluded premature neonates born at $<37$ weeks gestational age and children with cardiac, neurological or respiratory conditions who would not tolerate inadequate sedation. How this was determined is unclear and is an important confounder in this study, as only 132 (12\%) of 1059 patients screened over a 5 -year period could be assigned to the intervention arm of either DSI + PS $(n=66)$ or PS $(n=63)$. Unfortunately, the study was terminated prior to the expected recruitment total of 200 patients because of low recruitment rates, making the interpretation of the results - using a primary endpoint of number of ventilator-free days at day 28 - difficult.

Their results showed no difference in ventilator-free days; 24.0 days in both groups. The median ICU and hospital length of stay were similar in both groups: DSI + PS 6.9 days (interquartile range (IQR) 5.2 - 11.0) v. PS 7.4 days (IQR 5.3 - 12.8) $(p=0.47)$, and DSI + PS 13.3 days (IQR 8.6 - 26.7) v. PS 15.7 days (IQR 9.3 - 33.2) $(p=0.19)$. Mortality at 30 days was higher in the DSI + PS group than in the PS group (6/66 v. 0/63, respectively, $p=0.03$ ), though no causal relationship with the intervention could be established. The median cumulative midazolam dose did not differ: DSI + PS $14.1 \mathrm{mg} / \mathrm{kg}$ (IQR 7.6 - 22.6) v. PS $17.0 \mathrm{mg} / \mathrm{kg}$ (IQR 8.2 - 39.8) ( $p=0.11$ ).

This surprising finding of increased mortality could not be explained by the authors but is a signal of possible harm as a result of drug interruption in children, in contrast to the experience among adults. Thus, DSI in the paediatric ICU is currently not recommended, as the adverse effects are well known and should be minimised and avoided.

\section{R Masekela}

Paediatric Pulmonologist and Head of Department of Maternal and Child Health, Nelson R Mandela School of Clinical Medicine, University of KwaZuluNatal, Durban, South Africa

\section{Vet NJ, de Wildt SN, Verlaat CW, et al. A randomized controlled trial of daily sedation interruption in critically ill children. Intensive Care Med 2016;42(2):233- 244. DOI:10.1007/s00134-015-4136-z}

S Afr Respir J 2016;22(2):48. DOI:10.7196/SARJ.2016.v22i2.90

\author{
The South African Respiratory Journal \\ PO Box 13725 \\ Mowbray \\ 7705
}

Any correspondence to the Editor should be sent to the same address or via email to: sarj@iafrica.com

The website of The South African Thoracic Society can be found at www.pulmonology.co.za 


\title{
Should sedation holidays be given in the paediatric intensive care unit?
}

Although sedation interruption in the intensive care unit (ICU) is recommended in the literature for adults, its place in paediatrics has been much debated. The arguments for not using sedation interruption protocols include the danger of extubation, ventilatorassociated pneumonia, safety, comfort and, most importantly, synchronisation with the ventilator. There are also conflicting reports and a poor evidence base for the use of this strategy, with only two published randomised controlled trials comparing daily sedation interruption and protocolised sedation. An article by Vet et al. ${ }^{[1]}$ presented the results of a multicentre, randomised controlled trial in the Netherlands on this much-debated issue. The aim of their study was to compare daily sedation interruption plus protocolised sedation (DSI + PS) with protocolised sedation (PS) only in critically ill children. They excluded premature neonates born at $<37$ weeks gestational age and children with cardiac, neurological or respiratory conditions who would not tolerate inadequate sedation. How this was determined is unclear and is an important confounder in this study, as only 132 (12\%) of 1059 patients screened over a 5 -year period could be assigned to the intervention arm of either DSI + PS $(n=66)$ or PS $(n=63)$. Unfortunately, the study was terminated prior to the expected recruitment total of 200 patients because of low recruitment rates, making the interpretation of the results - using a primary endpoint of number of ventilator-free days at day 28 - difficult.

Their results showed no difference in ventilator-free days; 24.0 days in both groups. The median ICU and hospital length of stay were similar in both groups: DSI + PS 6.9 days (interquartile range (IQR) 5.2 - 11.0) v. PS 7.4 days (IQR 5.3 - 12.8) $(p=0.47)$, and DSI + PS 13.3 days (IQR 8.6 - 26.7) v. PS 15.7 days (IQR 9.3 - 33.2) $(p=0.19)$. Mortality at 30 days was higher in the DSI + PS group than in the PS group (6/66 v. 0/63, respectively, $p=0.03$ ), though no causal relationship with the intervention could be established. The median cumulative midazolam dose did not differ: DSI + PS $14.1 \mathrm{mg} / \mathrm{kg}$ (IQR 7.6 - 22.6) v. PS $17.0 \mathrm{mg} / \mathrm{kg}$ (IQR 8.2 - 39.8) ( $p=0.11$ ).

This surprising finding of increased mortality could not be explained by the authors but is a signal of possible harm as a result of drug interruption in children, in contrast to the experience among adults. Thus, DSI in the paediatric ICU is currently not recommended, as the adverse effects are well known and should be minimised and avoided.

\section{R Masekela}

Paediatric Pulmonologist and Head of Department of Maternal and Child Health, Nelson R Mandela School of Clinical Medicine, University of KwaZuluNatal, Durban, South Africa

\section{Vet NJ, de Wildt SN, Verlaat CW, et al. A randomized controlled trial of daily sedation interruption in critically ill children. Intensive Care Med 2016;42(2):233- 244. DOI:10.1007/s00134-015-4136-z}

S Afr Respir J 2016;22(2):48. DOI:10.7196/SARJ.2016.v22i2.90

\author{
The South African Respiratory Journal \\ PO Box 13725 \\ Mowbray \\ 7705
}

Any correspondence to the Editor should be sent to the same address or via email to: sarj@iafrica.com

The website of The South African Thoracic Society can be found at www.pulmonology.co.za 